\title{
An Unusual Cause of Keratitis
}

\author{
Saroj Gupta, ${ }^{1}$ Atul Aher ${ }^{2}$ \\ ${ }^{1}$ Department of Ophthalmology, and ${ }^{2}$ Department of Microbiology, People's College of \\ Medical Sciences and Research Centre, Bhopal, India
}

This report is of an unusual case of keratitis in a young healthy man who presented with minor trauma to his left eye. At examination, a characteristic ring infiltrate was seen in the centre of the cornea. Normal saline wet mount preparation of the corneal scraping showed unusual motile trophozoites of a parasite. Culture on non-nutrient agar with Escherichia coli overlay was negative. The patient was treated with topical neomycin, polymyxin $B$, and bacitracin eye drops and responded well to treatment.

Key words: Acanthamoeba, Cell culture techniques, Flagella, Keratitis, Parasite, Trophozoites

Asian J Ophthalmol. 2011;12:177-8.

\section{Introduction}

The unique structure of the human eye and exposure of the eye directly to the environment renders it vulnerable to a number of uncommon infectious diseases caused by fungi and parasites. There are many parasites that can affect the eye, including protozoans such as Toxoplasma spp, Acanthamoeba spp, Microsporidia spp, Giardia spp, and Rhinosporidia spp. The timely identification and treatment of the involved microorganisms are paramount in preventing visual loss. ${ }^{1}$

Acanthamoeba keratitis has been reported previously, ${ }^{2-4}$ and this report describes an unusual case of keratitis caused by a motile parasite following a minor eye injury.

\section{Case Report}

A 31-year-old man presented to the Department of Ophthalmology, People's College of Medical Sciences and Research Centre, Bhopal, India, in 2008 with pain, redness, and watering in his left eye for 5 days. He had a history of minor trauma (foreign body) in the left eye while working in the fields. Immediately after the incident, he washed his eye with stagnant dirty water.

At examination, the right eye was normal. The left eye showed an epithelial defect in the centre of the cornea, measuring $3.0 \mathrm{x}$ $2.5 \mathrm{~mm}$, with a prominent ring infiltrate surrounding the defect. The corneal infiltrate extended to the anterior stroma (Figure 1). Aseptic corneal scraping was performed and normal saline wet-mount

Correspondence: Dr Saroj Gupta, M-6 Windsor Estate Phase II, Chuna Bhatti Kolar Road, Bhopal, Madhya Pradesh 462016, India. Tel: (91 099) 2655 0364;

E-mail: sarojini94@yahoo.co.in
Figure 1. Ring infiltrate in the centre of the cornea.

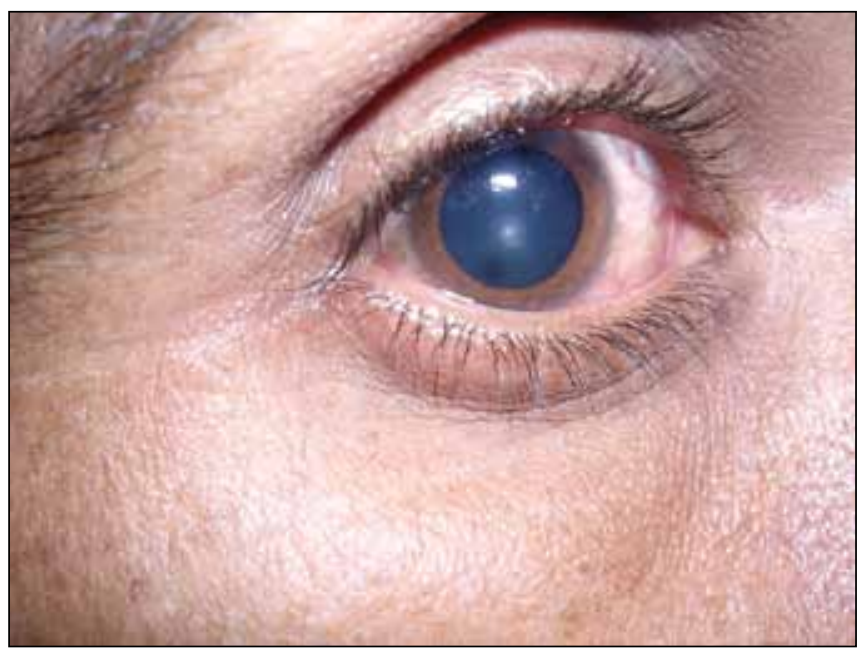

examination showed unusual motile trophozoites (Figure 2). The rapid shooting movement of the organism was suggestive of a flagellated protozoan. Potassium hydroxide $10 \%$ mount did not show hyphae or other elements resembling fungus. The material was inoculated on non-nutrient agar overlaid with Escherichia coli (monoxenic culture) for free-living amoebas and no growth was observed 2 weeks after incubation.

The patient started a regimen of topical neomycin, polymyxin B, and bacitracin eye drops (Neosporin ${ }^{\circledR}$, Burroughs Wellcome Inc, Kirkland, Canada) 1 hourly, clotrimazole 1\% eye drops 2 hourly, and atropine $1 \%$ eye drops twice daily.

The cornea improved within 1 week of therapy. The frequency of administration of drops was gradually tapered. At the 6-week follow-up, the cornea was clear with no evidence of subepithelial opacification. 
Figure 2. Trophozoite in a normal saline wet-mount preparation of corneal scraping (original magnification, $x$ 400).

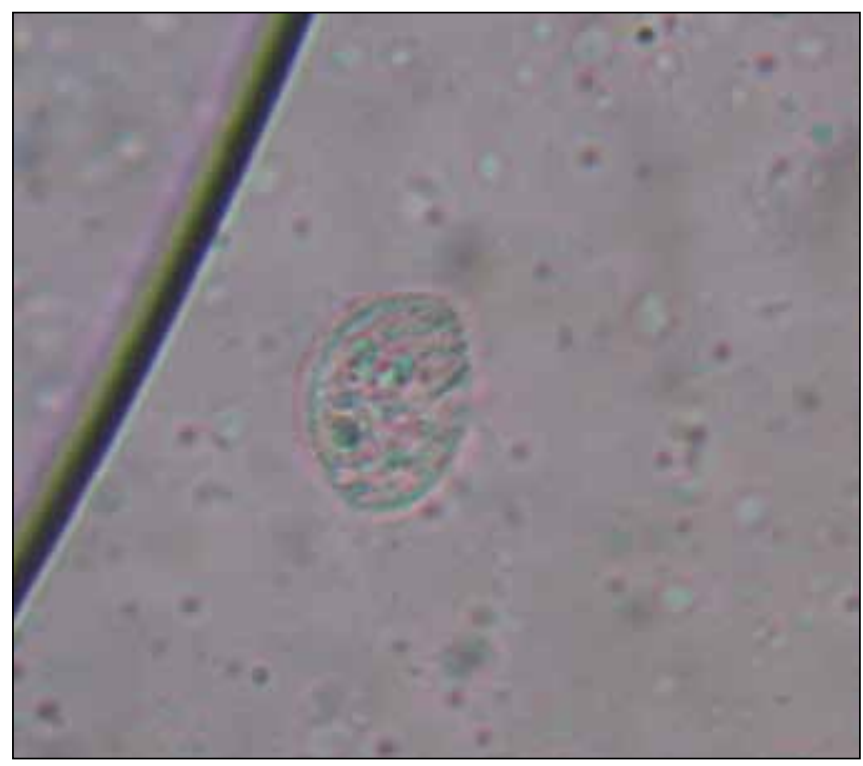

\section{Discussion}

There have been several reports from different parts of the world describing the spectrum of microbial keratitis..$^{5-8}$ The causative agents described in these reports have all been bacteria, fungi, and parasites. Among the parasites, Acanthamoeba has been reported frequently. ${ }^{2-4}$

The rapid shooting motility of trophozoites in saline wet mount suggested a flagellated protozoan such as Giardia spp, Chilomastix spp, or Naegleria spp. The motility of the organism was too rapid to diagnose it as a free-living amoeba. As there was no growth in nonnutrient agar, organisms such as Acanthamoeba spp and Naegleria spp were excluded. The typical motility of trophozoites on fresh examination is the key to diagnosis, so normal saline preparation of corneal scraping should be done to exclude motile parasites. Newer techniques such as confocal microscopy for in vivo identification of micro-organisms and polymerase chain reaction analysis can be used for rapid diagnosis. ${ }^{9,10}$

The current medical treatment of Acanthamoeba keratitis includes 1 or more of the following preparations: neomycin, polymixin-B, and bacitracin eye drops 1 to 2 hourly; clotrimazol $1 \%$ eye drops 2 hourly; propamide isethionate $0.1 \%$ eye drops 1 to 2 hourly; chlorhexidine digluconate $0.02 \%$ eye drops 1 to 2 hourly; and polyhexamethylene biguinide $0.02 \%$ eye drops 1 to 2 hourly., ${ }^{3,4}$

Propamide isethionate and polyhexamethylene biguinide were not available for this patient, so a combination of Neosporin and clotrimazole eye drops was used. Surprisingly, the response to treatment was good.

A high level of clinical suspicion and wet-mount examination of specimens from infected tissue are essential to aid in the rapid diagnosis of unusual forms of keratitis.

\section{Acknowledgements}

With grateful thanks to Dr Savitri Sharma, Associate Director and Head, Microbiology Services, LVPEl network, LV Prasad Eye Institute, Patia, Bhubaneswar, Orissa, India, for her expert opinion on the video of the corneal scrapping, and to Dr Omar M Amin, Director, Parasitology Center, Inc. (PCI), Scottsdale, Arizona, USA, for his expert opinion in this case.

\section{References}

1. Klotz SA, Penn CC, Negvesky GJ, Butrus SI. Fungal and parasitic infections of the eye. Clin Microbiol Rev. 2000;13:662-685.

2. Devamani F, Gnanaselvan J, Anandakannan K, Sridhar N, Sundararaj T. Studies on the prevalence of Acanthamoeba keratitis in and around Chennai. Indian J Med Microbiol. 1998:16:152-3.

3. Sharma S, Shrinivasm M, George G. Diagnosis of Acanthamoeba keratitis - a report of four cases and review of literature. Indian $J$ Ophthalmol. 1990;38:50-6.

4. Manikandan P, Bhaskar M, Revathy R, John RK, Narendran V, Panneerselvam K. Acanthamoeba keratitis - a six year epidemiological review from a tertiary care eye hospital in South India. Indian J Med Microbiol, 2004;22:226-30.

5. Gopinathan U, Sharma S, Garg P, Rao GN. Review of epidemiological features, microbiological diagnosis and treatment outcome of microbial keratitis: Experience over a decade. Indian J Ophthalmol. 2009;57:273-9.

6. Hagan M, Wright E, Newman M, Dolin P, Johnson G. Causes of suppurative keratitis in Ghana. Br J Ophthalmol. 1995;79:1024-8.

7. Upadhyay MP, Kamacharya PC, Koirala S, at al. Epidemiological characteristics, predisposing factors, and etiologic diagnosis of corneal ulceration in Nepal. Am J Ophthalmol. 1991;111:92-9.

8. Liesegang TJ, Forster RK. Spectrum of microbial keratitis in south Florida. Am J Ophthalmol. 1980;90:38-47.

9. Mathers WD, Nelson SE, Lane JL, Wilson ME, Allen RC, Folberg R Confirmation of confocal microscopy diagnosis of Acanthamoeba keratitis using polymerase chain reaction analysis. Arch Ophthalmol, 2000;118:178-83.

10. Boggild AK, Martin DS, Lee TY, Yu B, Low DE. Laboratory diagnosis of amoebic keratitis: comparison of four diagnostic methods for different types of clinical specimens. J Clin Microbiol. 2009;47: 1314-8. 